\section{Applied physics}

\section{Quantum cascade laser lives on the edge}

\section{Sunil Mittal \& Edo Waks}

Devices known as quantum cascade lasers produce useful
terahertz radiation, but are typically highly sensitive to
fabrication defects. This limitation has now been overcome
using a property called topological robustness. See p.246

Electromagnetic waves with frequencies in the terahertz range $(300 \mathrm{GHz}$ to $10 \mathrm{THz}$ ) have applications in many areas, from imaging and security screening to the atmospheric and biological sciences. Semiconductor devices called quantum cascade lasers (QCLs) provide the most compact and efficient way to generate terahertz radiation. In QCLs, electrons cascade down in energy through a series of discrete quantum energy levels, emitting a photon at each step ${ }^{1}$. But, as with all compact semiconducting lasers, QCLs are notoriously sensitive to fabrication imperfections, which results in device-to-device variability of the laser output frequency. Now, on page 246, Zeng et al. ${ }^{2}$ report the realization of a terahertz QCL that is insensitive to such disorder. This achievement opens the door for terahertz lasers and optoelectronics that have unprecedented stability and fabrication reproducibility.

Lasers use a process known as optical feedback to build up light intensity and stimulate electrons to emit photons. A common way to introduce this feedback uses a structure called an optical cavity, which is typically composed of mirrors that reflect the emitted light back into the device. Compact lasers, however, use more-complex structures such as photonic crystals - materials that have a periodically varying refractive index. If this periodicity is carefully engineered, photonic crystals can be used to reflect light waves of only the desired frequency, and so achieve lasing ${ }^{3}$. But this approach is highly sensitive to disorder, because any imperfections in the photonic crystal cause reflections that result in waves of unwanted frequencies. These compete with the desired waves, leading to unstable light intensity and poor laser efficiency.

In the past few years, 'topological' photonic structures have emerged as a way to make against fabrication imperfections. photonic devices that are insensitive to disorder. This area of research originated from concepts developed in condensed-matter physics. Over the past two decades, condensed-matter physicists have been able to use the mathematical descriptions of symmetries and topology to characterize different forms of matter. Of particular relevance to the current work are exotic materials known as topological insulators ${ }^{4}$.

As the name suggests, these materials are insulators - that is, they do not conduct electricity in their interior. However, they host conducting electronic states at their boundaries. Such edge states can carry current in only one direction and are therefore robust against disorder that would otherwise scatter charge carriers. This robustness of edge states is a manifestation of the overall topological properties of the material. Topological insulators are so insensitive to disorder that they were previously used to define the unit of resistance: the ohm.

Although topological physics originated in the field of electronics, it has begun to inspire photonics ${ }^{5}$. Disorder and scattering are even more problematic in optics than in electronics, because photons exhibit strong interference effects that can lead to complicated, difficult-to-control laser behaviour. Translating topological protection into the optical domain opens up the possibility of making robust optical systems. In particular, topological lasers can emit light in a way that is robust against scattering and other consequences of imperfections. But previous realizations of topological lasers ${ }^{6-8}$ have operated at frequencies above the terahertz range.

Zeng and colleagues overcame this limitation by incorporating topological protection into a QCL. To achieve this, they used a topological model known as the valley Hall effect, which relies on breaking the spatial-inversion symmetry of a crystal lattice ${ }^{9}$ (its symmetry under the combination of $180^{\circ}$ rotation and a mirror reflection). Specifically, the authors used a gallium arsenide-aluminium gallium arsenide substrate as the gain material the medium in which light is amplified. This substrate contained layered semiconductor structures called quantum wells that were designed to support quantum cascade lasing.

Theauthors drilled a triangular lattice of holes in the gain material (Fig. 1). The symmetries of

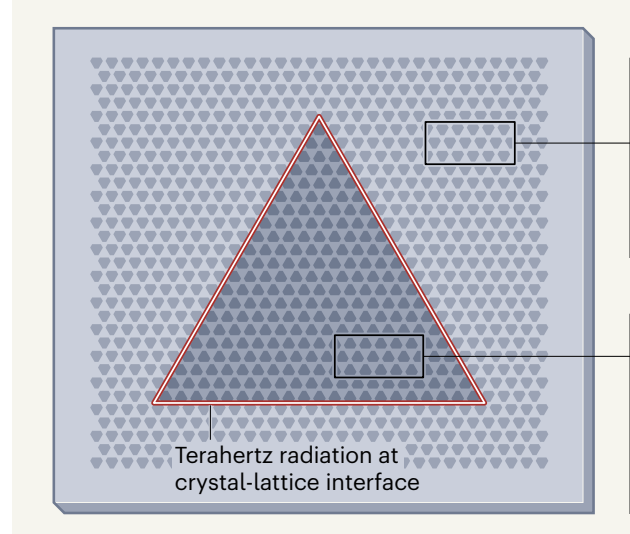

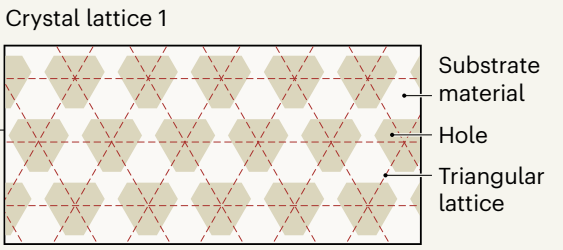

Crystal lattice 2

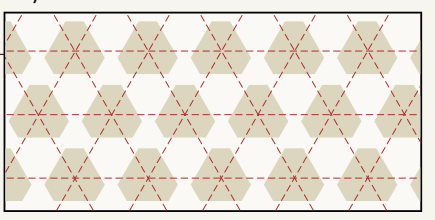

Figure 1 | Design of a topological laser. Zeng et al. ${ }^{2}$ have made a laser in which terahertz radiation is emitted from the interface between two triangular crystal lattices that consist of quasi-hexagonal holes in a substrate material. The crystal lattices are topologically inequivalent because the orientation of the holes is flipped in one lattice with respect to the other, and this leads to the emergence of exotic photonic states called edge states at the crystal-lattice interface. The topological nature of these edge states renders the laser robust 
this lattice resulted in the emergence of two valleys in the energy-momentum band structure - the relationship between the energy and momentum of photons in the material. The authors made the holes quasi-hexagonal so that they broke the spatial-inversion symmetry of the lattice and rendered the two valleys topologically inequivalent. This led to the formation of topological edge states at the interface between two such crystal lattices in which the orientation of holes (and valleys) was flipped in one lattice with respect to the other.

Zeng and co-workers used these topological edge states to design and make a robust ring resonator (a type of optical cavity that traps light at certain 'resonance' frequencies) in the form of a triangle (Fig. 1). It is this triangular cavity that, along with the light amplification from the substrate material, forms a topological laser. The laser produces light of many frequencies that are separated by similar frequency gaps. These frequencies correspond to the resonance frequencies of the triangular cavity and fall within the frequency range of the QCL gain material.

The authors measured light emission from different points along the perimeter of the cavity and discovered that the emission at each point had the same resonance frequencies. This indicates that these waves travelled through the length of the cavity, traversing the sharp $\left(60^{\circ}\right)$ bends at the corners of the triangle. Furthermore, Zeng et al. found that the lasing frequencies did not change when they introduced defects, in the form of extra holes, around the cavity, demonstrating the robustness of the QCL.

Another key feature of this laser is that energy is 'pumped' into the device electrically. Previous topological lasers ${ }^{6-8}$ have been optically pumped, which means that they require a second laser source to drive the topological laser to generate light. This pumping scheme severely limits practical applications. However, similar to many commonly used lasers (such as laser pointers), Zeng and colleagues' QCL can be directly driven by an electrical current, allowing it to be powered, in principle, by a battery or a wall outlet, rather than by another laser.

Robustness against defects and disorder is one defining characteristic of topological physics, but another important feature is a type of asymmetry called chirality. In particular, in the valley Hall effect, the two valleys are associated with photons of opposite circular polarization in the plane of the material. If right-circularly polarized photons travel to the left, then left-circularly polarized ones would travel to the right. Realizing this chirality represents a crucial future step towards terahertz topological lasers in which light waves flow around a ring resonator in only one direction. The chirality could be incorporated either by explicitly breaking time-reversal symmetry (a symmetry in which reversing the direction of light waves is equivalent to running time backwards) or by introducing directional light amplification in the cavity.

Zeng and co-workers' results pave the way for studying topology in a previously inaccessible part of the electromagnetic spectrum. One area of great interest for future research is the application of other topological models, such as exotic (higher-order) topological insulators, to make robust terahertz lasers that have other geometries. For example, these lasers could emit light at the corners, rather than at the edges, of a triangular cavity.

Another fascinating prospect is the exploration of non-Hermitian (open) physical systems at terahertz frequencies, in which the presence of light amplification and loss can lead to the emergence of features such as parity-time symmetry (symmetry under the combination of a mirror reflection and time reversal) and exceptional points (spectral features that correspond to coalescing resonances) ${ }^{10}$. The realization of topological photonics in the terahertz range could therefore serve as a catalyst for the development of practical devices, and also enable a better fundamental understanding of topological physics and complex (nonlinear) optoelectronics.

Sunil Mittal and Edo Waks are at the Joint

Quantum Institute, University of Maryland,

College Park, Maryland 20742, USA.

e-mails: mittals@umd.edu;

edowaks@umd.edu

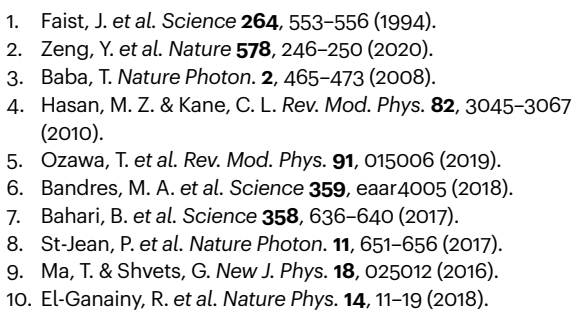

Structural biology

\section{Thorsten Althoff \& Jeff Abramson}

Unlike many sugar-transporting proteins, a transporter in one species of malaria parasite can import several types of sugar equally effectively, aiding the parasite's survival. The structure of this protein reveals the reason for its versatility. See p.321

Most cases of malaria are caused by the protozoan parasite Plasmodium falciparum ${ }^{1}$. Given that there are more than 400,000 malaria-associated deaths annually, and that $P$. falciparum is constantly evolving to resist pharmacological therapies, opportunities for developing drugs that target this organism must be continuously explored. A protein called the $P$. falciparum hexose transporter 1 (PfHT1) has a proclivity for scavenging sugars from an infected host's red blood cells to improve the parasite's chances of survival in these cells, and is therefore a drug target. On page 321, Qureshi et al. ${ }^{2}$ describe the 3D structure of PfHT1, and identify a mechanism that couples the docking of a sugar in the PfHT1 binding site to the process by which sugars are gated through the protein. This coupling facilitates the protein's substrate promiscuity - that is, its ability to transport a wide range of sugar molecules effectively, a feature that gives the parasite a distinct survival advantage.

Transporter proteins shuttle substrate molecules across the otherwise impermeable lipid bilayer of the cell membrane. The functional and dynamic properties of these membrane-embedded proteins are fundamentally related to their 3D structures, which are modulated at the atomic level over a broad range of timescales. Membrane transporters use the alternating-access mechanism for gating ${ }^{3}$, in which access to the substrate-binding site switches from one side of the membrane to the other (Fig. 1).

The development of methods for determining the structures of membrane proteins in the past few years has produced near-complete pictures of the translocation mechanisms of several classes of transporter - that is, the global rearrangements that the proteins undergo during translocation cycles of substrate binding, transport and release have been visualized at atomic resolution. Intuitively, the substrate specificity of transporters has generally been found to depend on the amino-acid residues at the binding 